\title{
A Tampered Image Detecting Method Based on Wavelet Analysis
}

\author{
Wenqing Bu, Ming Xu, and Ning Zheng
}

\begin{abstract}
Contrast enhancement operation is often used to highlight some information, weaken or remove some unwanted information in tampered images. In this paper, a tampered image detecting method is proposed based on wavelet analysis for specific fingerprints left by contrast enhancement operation in image. Firstly, the RGB color space of image was converted into YCbCr color space, and the $\mathrm{Y}$ monochrome channel image was extracted; secondly, the normalized energy was calculated in the wavelet details sub-bands after wavelet transform of image's pixel value histogram of the component; finally, the tampered image was identified according to the normalized energy. The results of the experiment show there is an obvious distinction between tampered and unaltered sub-blocks divided. Furthermore, the contrast experiment results about wavelet transform and Fourier transform show that the former is better than the latter in both accuracy and time complexity.
\end{abstract}

Index Terms-Contrast enhancement, fingerprints, histogram, wavelet transform

\section{INTRODUCTION}

In recent years, digital images have come to play an important role in news media, law enforcement, and military applications. Therefore it is necessary to address the digital blind forensics techniques for verifying an image's authenticity. "Please delete photos taken on 15 July and sent on 16 July (2011) by north Korea's central news agency (kcna). The photos were suspected of using digital technology with PS," The Associated Press questioned the authenticity of these pictures which presented DaTong River, where water level had soared, highway was overflowed by the river's waters and several north Korea's residents were walking in the water up to the knees, suffered from days of heavy rain. South Korea's unification ministry officials said such tampering seemed to make north Korea in the exaggerated situation to acquire aid of the international community. In general, the event reflects that detecting tampered image is meaningful.

It is unacceptable that the water parts with different colors in the pictures sent by kcna are actually projection that forgers haven't had time to delete which left much obvious false traces. At this moment, appending contrast

Manuscript received July 30, 2011; revised October 11, 2011. This work was supported by NSFC (No. 61070212 and 61003195), Natural Science Foundation of Zhejiang Province, China (No. Y1090114); the State Key Program of Major Science and Technology (Priority Topics) of Zhejiang Province, China (No 2010C11050; e-mail: nzheng@hdu.edu.cn).

Wenqing $\mathrm{Bu}$ and Ning Zheng are now with the Institute of Computer Application Technology, Hangzhou Dianzi University, Hangzhou, China, 310018(e-mail: bu wenqing@126.com).

Ming $\mathrm{Xu}$ is now with the Institute of Computer Application Technology, Hangzhou Dianzi University, Hangzhou, China, 310018(e-mail: mxu@hdu.edu.cn). enhancement globally or locally to the tampered image will enable a more natural mosaic image not only to retouch or conceal visual evidence of forgery, but also in an attempt to destroy forensically significant indicators of these tampering operations. Contrast enhancement belongs to the category of content-preserving manipulations [1] as well as resampling [2], compression [3], blur [4], [5] and sharpening [6] other than content-changing tampering such as splicing and copy-move [7], [8]. In brief, strengthening contrast enhancement detection is of great significance since the type of operations is also frequently used to merely change image perceptual quality in the general image processing.

Although skillful contrast enhancement forgeries leave no visible trace to ordinary viewers, they often contain underlying unique statistic fingerprints. M.C.Stamm and K.J.R.Liu [9] expected $H(\omega)$, the discrete Fourier transform (DFT) of an unaltered image's pixel value histogram served as contrast enhancement statistical fingerprint, to be a strongly low-pass signal. Monotonically increasing contrast enhancement mapping results in an increase in energy within the pixel value histogram. The increase in energy tends to be spread across the frequency spectrum. Consequently, energy introduced in the high frequency regions of $H(\omega)$ affords a clue for detection of images that have experienced contrast enhancement.

However, both accuracy and time complexity can be further improved. This paper extracts the image's Y monochrome channel in the $\mathrm{YCbCr}$ color model and uses wavelet analysis to detect the contrast enhancement operation. We do one-dimensional discrete wavelet transform (1D-DWT) and one-dimensional DFT (1D-DFT) of image's pixel value histogram respectively. For the wavelet analysis, we calculate the normalized measure of the energy of the wavelet details sub-bands. For the Fourier analysis, we calculate the normalized measure of the energy in the high frequency components of the pixel value histogram. Regardless of recognition rate of false images or time complexity, wavelet analysis is superior to Fourier analysis.

The rest of the paper is organized as follows. Section $\Pi$ surveys related work of the digital image forensics technology. In section III, we describe the key wavelet analysis methodology and Fourier analysis methodology. Section IV gives experimental results and discussions about the results. We conclude this paper in section $\mathrm{V}$.

\section{RELATED WORK}

The linear regression classifier has been exploited to suspect the authenticity of the image and detect false area 
operated by contrast enhancement [10]. [1] Focused on the blind detection of median filtering rely on the probability of zero values on the first order difference map in texture regions. Gradient aberration of the gray histogram and ringing artifacts around step edges were measured and employed to capture trails of sharpening operation in [6].

To the best of our knowledge, image enhancement alterations fall into many categories [1], [6] and the detection history involving contrast enhancement is not long. M.C.Stamm and K. J. R. Liu [9], [11], [12] have constructed a model for the histogram of an unaltered image, and took advantage of this model to detect artifacts left behind by alterations in the form of global and local contrast enhancement operations, obtaining $P_{d} \quad 0.99$ based on the $\mathrm{G}$ channel in the RGB color model. Apart from this, they were capable of estimating and reconstructing of a contrast enhancement mapping in [13]. [14] Identified and reconstructed gamma correction operations. To be undetectable by the existing contrast enhancement detectors, [15] developed anti-forensic techniques by which local random dithering was introduced into the design of contrast enhancement mapping.

Our contrast enhancement detecting approach is similar to M.C.Stamm and K.J.R.Liu based on the peak-gap artifacts of the pixel graylevel histogram [9]. The core idea of this paper is that a $1 \mathrm{D}$-DWT method is used to calculate the normalized measure of the energy generated in the high frequency regions of wavelet details sub-bands. The value within $\mathrm{Y}$ channel distinguishes the altered images and achieves a satisfactory outcome.

\section{Methodology}

\section{A. Contrast Enhancement Based on Y Channe}

A color image can be decomposed into Red (R), Green $(\mathrm{G})$, Blue (B) three monochrome images. Processing is usually done by each of these three images. In the $\mathrm{YCbCr}$ color model, however, people are more sensitive to the $\mathrm{Y}$ component (luminance) which approximates the brightness information. The RGB color model representation does not isolate color information from luminance. Hence, we consider using the $\mathrm{Y}$ component monochrome channel image in $\mathrm{YCbCr}$ color space to detect the contrast enhancement operation. The relationship between RGB and $\mathrm{Y}$ is as follows.

$$
Y=0.2990 R+0.5870 G+0.1140 B
$$

Contrast enhancement operation makes details outstanding, fuzzy or obscure. It can be viewed as pixel value mapping. Typical contrast enhancement curves are in logarithmic form, exponential form, etc. For example, the power law transformation

$$
y=255\left(\frac{x}{255}\right)^{\gamma}
$$

is used in [9]. Our manner discusses the contrast enhancement processing by the mapping

$$
Y=\operatorname{imadjust}(X,[\text { low high }],[\text { bottom top }], \text { gamma })(3)
$$

to the original images. It indicates the image $X$ is adjusted to the image $Y$. [low high] is the gray range of the original image to transform. [bottom top] specifies the scope of the transformed gray. gamma decides to improve the low gray or enhance the high gray in the process of input image to the output image. The gray transformation function of imadjust is defined by (4) and simulated in Fig. 1.

$y=\left\{\begin{array}{cc}\text { bottom } & x \leq \text { low } \\ \left(\frac{\text { top }- \text { bottom }}{\text { high }- \text { low }}\right)^{\text {gamma }}(x-\text { low })^{\text {gamma }} & \text { else } \\ \text { top } & x \geq \text { high }\end{array}\right.$

The calibration curve is depicted in Fig. 1. When gamma $<1$, the image is lightened demonstrated in Fig. 2.

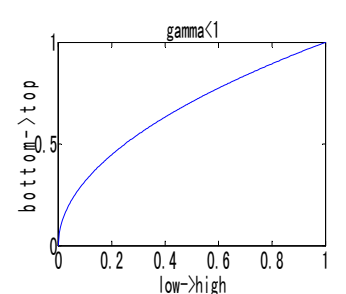

(a)

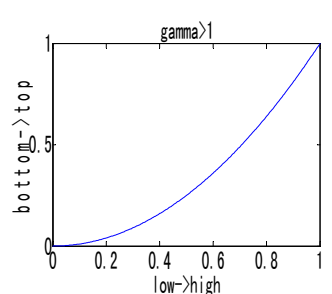

(b)
Fig. 1. The calibration curve (a) gamma $<1$ and (b) gamma $>1$.

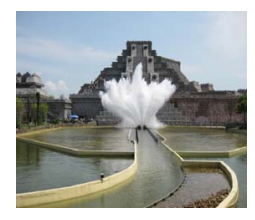

(a)

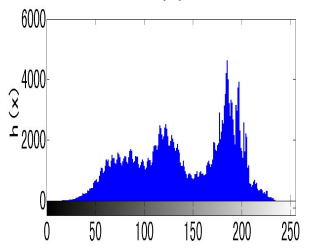

(c)

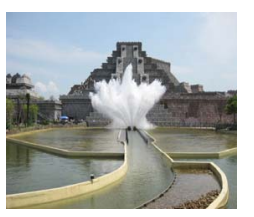

(b)

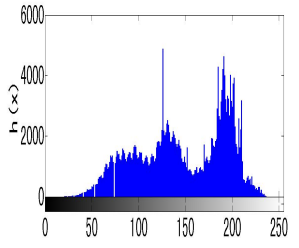

(d)
Fig. 2. The original (a), tampered (b) image and their pixel value histograms (c), (d).

Fig. 2 shows the histograms of an unaltered and the image that has undergone global contrast enhancement. Compared to original image, the adjusted image (b) is brighter. The peaks and gaps suddenly appear in Fig. 2(d). We describe the unaltered digital image's pixel value histogram as an interpolatably connected function. None of the original histograms contain sudden zeros or impulsive peaks. Individual histogram values should not greatly from the histogram's envelope [9]. Thus, the sudden peaks and gaps introduced into image's pixel value histogram $h(x)$ as a product of contrast enhancement lead to the addition of the energy in the high frequency components of the pixel value histogram as can be seen in Fig. 2(d) and Fig. 3(b). 


\section{B. Wavelet Analysis Method}

For the image signals, we are interested in the edge (local, high frequency) information. Standard Fourier transform, for a smooth and global signal, is either in the time domain or in the space domain. To solve the problem of time-frequency localization, we can add a sliding "time window $\operatorname{win}(x)$ " for Fourier transform, called the short time Fourier transform (STFT). However, since the win $(x)$ function is fixed, the time and frequency resolution of the STFT is fixed. Consequently, we adopt DWT which has the ability of analyzing both high frequency and low frequency of the signal at the same time. In other words, wavelet transform has a mutliresolution property in the time and frequency domains which overcomes the resolution problem in other transforms. Furthmore, wavelet time-frequency window changes with the center of the window, the window's width and the bandwidth also change. The function to wavelet decomposition is divided into many parts. Low frequency part is the broad contours of the function and high frequency part is the details.

The 1D-DWT of the signal $f(x)$ is defined as

$$
W_{f}(m, n)=\int_{-\infty}^{\infty} f(x) \psi_{m, n}^{*}(x) d x
$$

$\psi_{m, n}^{*}(x)=a_{0}^{-0.5 m} \psi\left(a_{0}^{-m} x-n b_{0}\right)$. where $\psi_{m, n}^{*}(x)$ is the mother wavelet. ${ }^{*}$ denotes complex conjugation. $W_{f}(m, n)$ is the calculated DWT coefficients which refers to the closeness of the signal to the wavelet at a particular scale. The DWT is for continuous dilation parameter $a_{0}^{m}$ and continuous translation parameter $n a_{0}^{m} b_{0}$, but not for the coordinate variables $x$.

There are a great many wavelet functions. For instance, like all wavelet transforms, the Daubechies wavelet $\mathrm{db} 1$ with $a_{0}=2$ and $b_{0}=1$ (5) transform decomposes a discrete signal into two subsignals of half its length. One subsignal is a running average or trend; the other subsignal is a running difference or fluctuation. The $\mathrm{dbl}$ wavelet transform is conceptually simple with asymmetric, orthogonal and biorthogonal properties as well as Haar wavelet. The db1 wavelet functions is defined as

$$
\psi_{d b 1}=\left\{\begin{array}{cc}
1, & 0 \leq x \leq 0.5 \\
-1, & 0.5 \leq x \leq 1 \\
0, & \text { else }
\end{array}\right.
$$

Fig. 3 depicts the low frequency and high frequency coefficients extracted from 1D-DWT of image's pixel value histogram based on Y component monochrome channel.
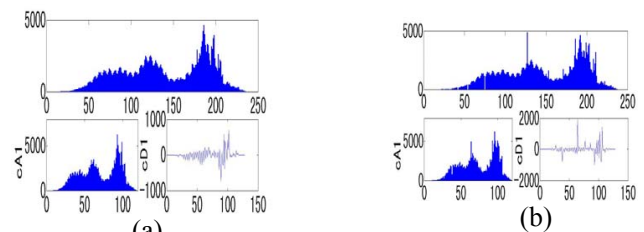

(b)

Fig. 3. Image's pixel value histogram (just above), low frequency coefficients (lower left) and high frequency coefficients (Lower right) after 1D-DWT of (a) the original image and (b) the image that has undergone contrast enhancement.

\section{Fourier Analysis Method}

Fourier analysis method, put forward by M.C.Stamm and K.J.R.Liu, demonstrates incisively and vividly in the sense of yielding large high frequency measurements indicative of contrast enhancement mapping fingerprints in [9], [11], [12]. Through understanding Fourier analysis method, we better grasp the essence of the images tampering by contrast enhancement. What's more, we cannot ignore histogram saturation effects exhibiting in unaltered images. This phenomenon can lead to break out with a impulse components peak in image's pixel value histogram of the original image which is perhaps mistaken as the fingerprint of a contrast enhancement mapping. In order to avoid causing wrong judgment on account of histogram saturation effects that produce large amounts of the high coefficients, we make image's pixel value histogram $h(x)$ a new changes to become $g(x)$.

$$
g(x)=w(x) h(x)
$$

$w(x)$ plays the role of the window clip function, defined as

$$
w(x)=\left\{\begin{array}{cc}
0.5-0.5 \cos (\pi x / n) & x \leq n \\
1 & \text { else } \\
0.5+0.5 \cos (\pi(x-255+n) / n) & x \geq 255-n
\end{array}\right.
$$

Here $n=4$ that refers to M.C.Stamm and K.J.R.Liu [9]. Fig. 4 shows the frequency domain representations of the histogram of an image before and after it has undergone contrast enhancement based on Y component monochrome channel.

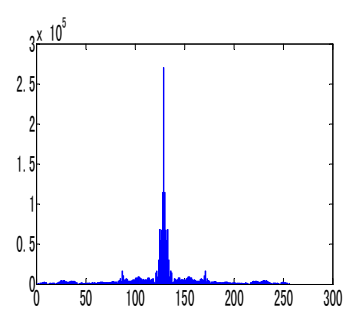

(a)

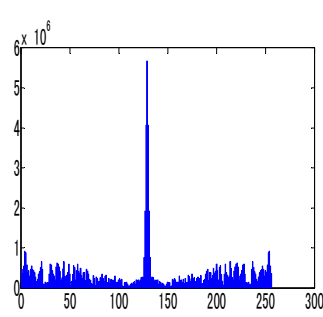

(b)
Fig. 4. The magnitude of the DFT of (a) the unaltered image's pixel value histogram in Fig. 2(c) and (b) the contrast enhanced image's pixel value histogram in Fig. 2(d).

Then, after $\operatorname{DFT}(w(x) h(x))$, we calculate the total energy from $N$ to 128 by (9). $N$ is the DFT 256 sampling points. $\mathrm{M}$ is the number of all the image's pixels.

$$
E_{d f t}=\frac{1}{M} \sum_{k=N}^{128} \operatorname{DFT}(w(x) h(x))
$$

\section{EVOLUTION AND EXPERIMENTS}

Image contrast enhancement includes gamma $<1$ and gamma $>1$ means. Contrast enhancement operation is generally used to make image lighter on the purpose. In view of this, this paper discussed tampering only 
when gamma $<1$. Correspondingly, the experiment was designed based on this adjustment. Tampering when gamma $>1$, we can obscure image details, hide some objects in the image where some objects are hard to recognize. This kind of situation comes to the conclusion that is consistent with that when gamma $<1$.

We downloaded 229 photos from many friends' album from the Internet (http://www.renren.com) and saved them as bitmaps. These pictures are different sizes, such as $3264 \times 2448$ pixels, $2560 \times 1920$ pixels, $600 \times 802$ pixels, $800 \times 600$ pixels, $600 \times 450$ pixels, $540 \times 720$ pixels, $100 \times 75$ pixels, etc. They were captured by various cameras or cell phones under different light conditions. A set of altered images were created by contrast enhancement operating by applying (3) with low $=0.3$, high $=0.7$, bottom $=0$, top $=1$ and gamma $=0.1,0.2,0.3,0.4,0.5,0.6,0.7,0.8$, 0.9 to pixel values of the $\mathrm{Y}$ component of these unaltered images. They were then saved as bitmaps, along with bitmaps versions of the original images, creating a testing database of 458 images as experiment object 1. Fig. 5 shows some of the original images.

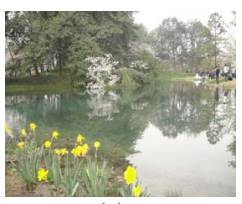

(a)

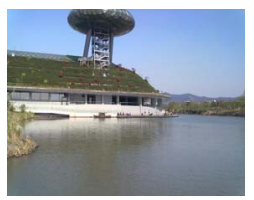

(b)

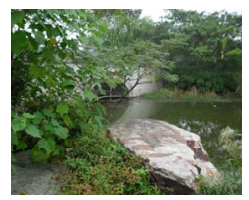

(c)

Fig. 5. Three original images of different sizes in different scenes taken by different cameras and cell phones.

Moreover, to evaluate the proposed contrast enhancement detection method, we conducted another experiment on the popular image dataset UCID [16]. We tampered the 244 TIFF images to create a testing database of 488 images as experiment object 2 in the light of steps as mentioned above.

\section{A. Wavelet Transform Detection of Globally Applied Contrast Enhancement}

Original image's pixel value histogram $h(x)$ is smooth. On the one hand, after 1D-DWT with the mother wavelet $\mathrm{db} 1$ according to (6), we calculated $E_{d w t}$, the normalized measure of the energy in wavelet domain of image's pixel value histogram according to (10). $i$ refers to the energy of wavelet details sub-bands. $M$ is the total number of pixels in the image On the other hand, we calculated $E_{d f t}$, the normalized measure of the energy from $N=112$ to 128 according to (7), (8), (9).

$$
E_{d w t}=\frac{1}{M} \sum_{i}|D W T(h(x))|
$$

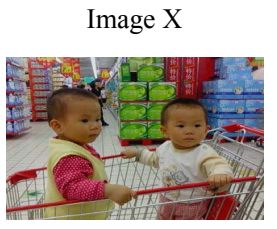

gamma $=0.9$

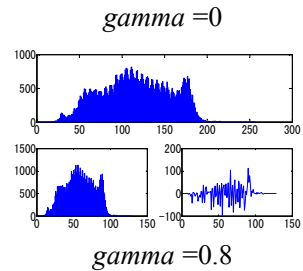

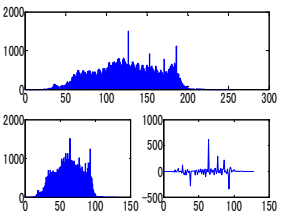

gamma $=0.7$

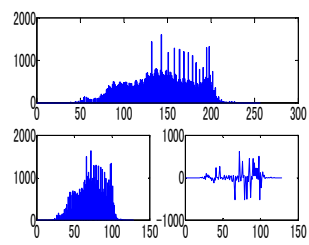

Fig. 6. The change trend diagram after wavelet transform of Image $X^{\prime}$ pixel value histograms under various contrast enhancement.

Fig. 6 gives the low frequency and high frequency coefficients extracted from 1D-DWT of Image X's pixel value histogram based on $\mathrm{Y}$ component monochrome channel. This image has been tampered with different degrees of contrast enhancement. From Fig. 6, we find image's pixel value histograms change after contrast enhancement. Along with more and more big contrast, the impulse components look more and more. In other words, the normalized energy in wavelet details sub-bands can yet be regarded as a more convenient and ideal feature for classification of tampering images. An overview of the proposed scheme is given in Fig. 7.

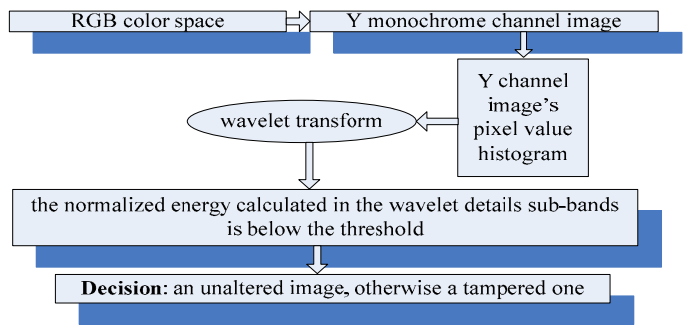

Fig. 7. A system for image contrast enhancement detection

In addition, under different contrast ( gamma $=0.9-0.1$ ), the average energy statistics trend of a great number of pictures is described in Fig. 8. Approximately, the original ones' is 0.0428 . Fig. 8 tells us no matter how big the contrast is, the normalized energy has been floating above the original image's. Similarly, this rule exists when gamma $>1$ as well as when gamma $<1$ because gamma $>1$ adjustment also belongs to the category of contrast enhancement tampering, just the image will be darken.

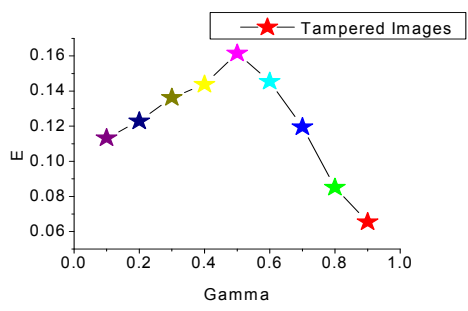

Fig. 8. Under different contrast ( gamma $=0.9-0.1$ ), the average energy statistics trend of a great number of pictures. 


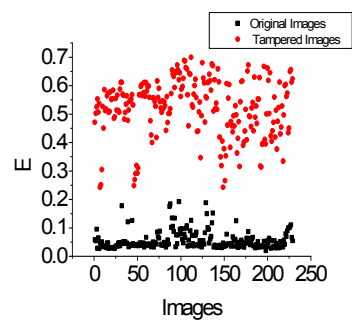

(a)

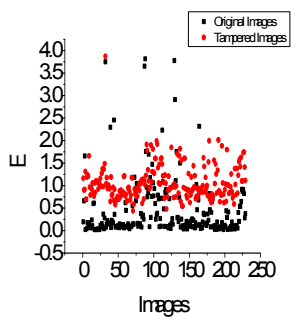

(b)

Fig. 9. Normalized measure of the energy in the high frequency components of the pixel value histogram in the (a) wavelet domain and (b) the frequency domain based on experiment object 1.

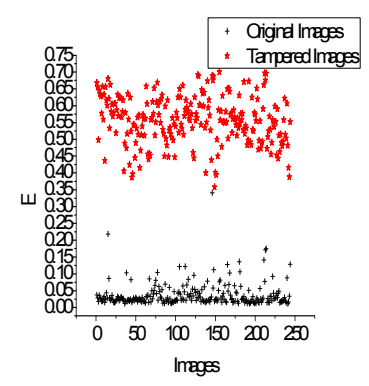

(a)

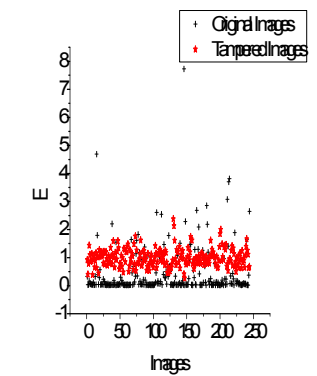

(b)

Fig. 10. Normalized measure of the energy in the high frequency components of the pixel value histogram in the (a) wavelet domain and (b) the frequency domain based on experiment object 2 ..

Fig. 9 and Fig. 10 show the results of performing global contrast enhancement detection on the $\mathrm{Y}$ color layer of images with gamma $=0.3$ based on experiment object 1 and 2 respectively. Black boxes/cross are the images unaltered Red solid circles/star are the tampered images. Both Fig. 9 and Fig. 10 depict an increase in energy after tampering. Clearly, recognition feature of fake pictures in Fig. 9(a) and Fig. 10(a) is more obvious than in Fig. 9(b) and Fig. 10(b) respectively.

In contrast, our wavelet analysis approach demonstrates a higher ability to identify false pictures than the Fourier analysis method under the circumstances of contrast enhancement with gamma $=0.1$ to 0.9. Some false affirmation rate weakens the performance of Fourier analysis. Troublesomely, a series of decision thresholds are required to train, such as the parameter $n=4$ in (8) and the parameter $N=112$ in (9). This is an extremely time-consuming process.

\section{B. Wavelet Transform Detection of Locally Applied Contrast Enhancement}

Compared with detection of global contrast enhancement, detection of local contrast enhancement is similar, but the unaltered and locally altered images were partitioned into square $119 * 119$ pixels in size to be processed separately. Only taking into account wavelet analysis, we calculated statistical $E_{d w t}$ of each block.

Fig. 11 provides (a) the original image and (b) the image altered by local contrast enhancement from 25 to 30 blocks based on the Y monochrome channel image. Fig. 11 (c) shows the results of performing localized contrast enhancement detection on the $\mathrm{Y}$ color layer of the image. Black boxes are blocks of the image unaltered. Red solid circles are blocks of the tampered image. When the black boxes and red solid circles overlap, the image has not been any changes. On the contrary, these blocks have experienced contrast processing. The latter displays the higher energy than the former from 25 to 30 blocks.

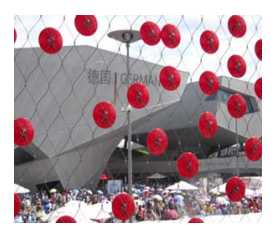

(a)

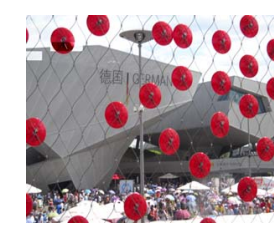

(b)

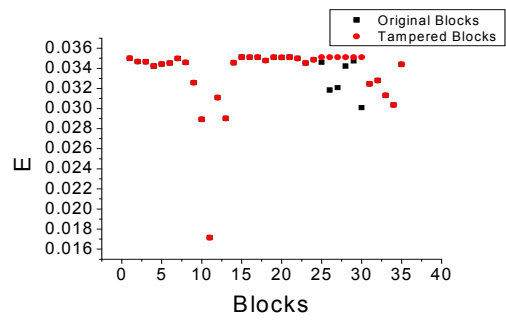

(c)

Fig. 11. (a) the original image, (b) the altered image, (c) normalized measure of the energy in the high frequency components of the image's pixel value histogram in the wavelet domain.

When detecting the alteration in [9], there is high false positive rate in the blue layer. Only with the combination of R, G, B three channels can they reach the desired results. Wavelet transform detection appears few false positive rate based on Y channel.

\section{CONCLUSIONS AND FUTURE WORK}

In this work, wavelet analysis has been proposed to detect the image unique fingerprints left in an image's pixel value histogram by globally and locally applied contrast enhancement based on $\mathrm{Y}$ channel. We identified these alterations by detecting the high frequency components in the wavelet domain and the frequency domain respectively. Simulation results indicated that wavelet analysis was better able to detect the application of contrast enhancement operation designed than Fourier analysis in the case of different contrast.

In the future, we will try to hunt for a mother wavelet which can more effectively identify tampered images. Besides, forensic detection of JPEG2000 image and digital image born in the printer are also worth studying. Our next research focus is to address the detection of noise globally and locally adding in the $\mathrm{YCbCr}$ color model of images. The further idea is to spread the noise detection to the forensics of anti-forensics of image manipulation.

\section{REFERENCES}

[1] G. Cao, Y. Zhao, R. Ni, L. Yu, and H. Tian, "Forensic detection of median filtering in digital images," in IEEE International Conference of Multimedia and Expo (ICME), July. 2010, pp. 89-94.

[2] A. C. Popescu and H. Farid, "Exposing digital forgeries by detecting traces of resampling," IEEE Transactions on Signal Processing, 2005, vol. 53, no. 2 , pp.758-767. 
[3] H. Farid, "Exposing digital forgeries from JPEG ghosts," IEEE Transactions on Information Forensics and Security, 2009, vol. 4, no. 1, pp.154-160.

[4] D. Hsiao and S. Pei, "Detecting digital tampering by blur estimation," 1st International Workshop on Systematic Approaches to Digital Forensic Engineering, Washington, 2005.

[5] G. Cao, Y. Zhao, and R. Ni, "Edge-based blur metric for tamper detection," Journal of Information Hiding and Multimedia Signal Processing, 2010, vol. 1, no. 1, pp.20-27.

[6] G. Cao, Y. Zhao, and R. Ni, "Detection of image sharpening based on histogram aberration and ringing artifacts," in IEEE International Conference Proceedings on Multimedia and Expo(ICME), New York, July. 2009, pp. 1026-1029.

[7] W. Chen, Y. Q. Shi, and W. Su, "Image splicing detection using 2-D phase congruency and statistical moments of characteristic function," SPIE Electronic Imaging: Security, Steganography, and Watermarking of Multimedia Contents, San Jose, CA, USA, 2007.

[8] S. Bayram, H. T. Sencar, and N. Memon, "An efficient and robust method for detecting copy-move forgery," in International Conf. on Acoustics, Speech and Signal Processing, Taipei, 2009.

[9] M. C. Stamm and K. J. R. Liu, "Forensic detection of image manipulation using statistical intrinsic fingerprints," IEEE Trans. Inf. Forensics and Security, Sep. 2010, vol. 5, no. 3, pp. 1556-6013.

[10] I. Avcibas, S. Bayram, N. Memon, M. Ramkumar, and B. Sankur, "A classifier design for detecting image manipulations," in Proc. ICIP, Oct. 2004, vol. 4, pp. 2645-2648.

[11] M. C. Stamm and K. J. R. Liu, "Blind forensics of contrast enhancement in digital images," in Proc. ICIP, San Diego, CA, Oct. 2008, pp. 3112-3115.

[12] M. C. Stamm and K. J. R. Liu, "Forensic detection of image tampering using intrinsic statistical fingerprints in histograms," in Proc. APSIPA Annual Summit and Conf., Sapporo, Japan, Oct. 2009.

[13] M. C. Stamm and K. J. R. Liu, "Forensic estimation and reconstruction of a contrast enhancement mapping," in IEEE International Conference Proceedings on Acoustics Speech and Signal Processing (ICASSP), March 2010, pp. 1698-1701.

[14] G. Cao, Y. Zhao, and R. Ni, "Forensic estimation of gamma correction in digital images," in Pro. ICIP, Sept. 2010, pp. 2097-2100.

[15] G. Cao, Y. Zhao, R. Ni, and H. Tian, "Anti-forensics of contrast enhancement in digital images," in Proceedings of ACM SIGMM Multimedia and Security Workshop, Sept. 2010, pp. 25-34.
[16] G. Schaefer and M. Stich, "UCID - An uncompressed colour image database," in Proc. SPIE, Storage and Retrieval Methods and Applications for Multimedia, 2003, vol. 5307, pp.472-480.

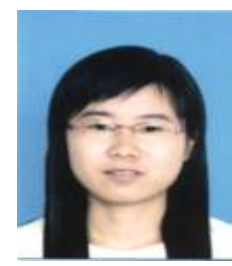

Wenqing Bu received the B.E. degree from Anhui Polytechnic University, China, in 2009. She is currently a graduate student for a Master's degree in Hangzhou Dianzi University, Hangzhou, China. Her research interests include digital image forensics and computer forensics.

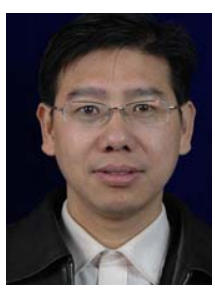

Ming Xu received the Ph.D. degree from Zhejiang University, China. He is currently a professor with Hangzhou Dianzi University, Hangzhou, China. His research interests include network security, digital forensics, content security, and information counterwork.

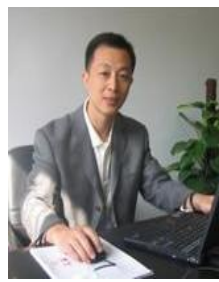

Ning Zheng received the Master degree from Zhejiang University, China. He is currently a professor and doctoral supervisor with Hangzhou Dianzi University, Hangzhou, China. His research interests include information management system and network information security. 